\title{
Coherent manipulation of quantum $\delta$-kicked dynamics: Faster-than-classical anomalous diffusion
}

\author{
Jiangbin Gong, Hans Jakob Wörner, and Paul Brumer \\ Chemical Physics Theory Group, Department of Chemistry, University of Toronto, Toronto, Canada M5S $3 H 6$
}

(Received 25 March 2003; published 18 August 2003)

\begin{abstract}
Large transporting regular islands are found in the classical phase space of a modified kicked rotor system in which the kicking potential is reversed after every two kicks. The corresponding quantum system, for a variety of system parameters and over long time scales, is shown to display energy absorption that is significantly faster than that associated with the underlying classical anomalous diffusion. The results are of interest to areas of both quantum chaos and quantum control.
\end{abstract}

DOI: 10.1103/PhysRevE.68.026209

PACS number(s): 05.45.Mt, 32.80.Qk, 05.60.Gg

The kicked-rotor system (KR) has long served as a paradigm for classical and quantum chaos [1]. Its atom optics realization [2] makes it possible to directly compare fundamental theoretical work with corresponding experimental studies. The KR is also of interest to a variety of other fields such as molecular physics $[3,4]$, condensed matter physics [5], and quantum information [6,7].

It is well known that quantum coherence suppresses classical chaotic (normal) diffusion. The suppression mechanism is well understood in terms of "dynamical localization" [1] in KR. By contrast, much less is known about quantum interference effects in the case of classical anomalous diffusion, a phenomenon induced by the fractal boundary between regular and chaotic regions in phase space. Of particular interest is the quantum dynamics of $\delta$-kicked systems where the underlying classical chaos coexists with transporting regular islands, e.g., the accelerator modes in KR [8-12]. In this case it is found that anomalous diffusion induced by transporting islands causes the early breakdown of quantumclassical correspondence (QCC) [8] and enhances fluctuations of dynamical localization length [9-11], and that quantum eigenstates in the semiclassical limit may ignore classical phase space structures [12].

Motivated by our recent studies on coherent manipulation of classically chaotic dynamics $[13,14]$, we consider here a modified kicked-rotor (MKR) model in which the kicking potential is reversed after every two kicks [15]. As shown below, this apparently slight modification of the KR system has a profound effect on the dynamics. First, it results in the appearance of transporting regular islands that are much larger and of a different nature than those previously observed. Second, and more importantly, we find a new phenomenon: The corresponding quantum system, for a variety of system parameters and over considerably long time scales, displays energy absorption that is significantly faster than that associated with the underlying classical anomalous diffusion. This result constitutes an important and intriguing aspect of quantum interference effects in classically chaotic systems. Furthermore, the drastic difference in quantum dynamics between KR and MKR is a demonstration of spectacular quantum control [16] over $\delta$-kicked systems, achieved here by periodically reversing or delaying the kicking field. These results are of broad theoretical and experimental interest.
The Hamiltonians for the KR and MKR systems are given by

$$
H^{K R}(\hat{L}, \theta, t)=\hat{L}^{2} / 2 I+\lambda \cos (\theta) \sum_{n} \delta\left(\frac{t}{T}-n\right)
$$

and

$$
H^{M K R}(\hat{L}, \theta, t)=\hat{L}^{2} / 2 I+\lambda \cos (\theta) \sum_{n} f(n) \delta\left(\frac{t}{T}-n\right) .
$$

Here $|f(n)|=1$ and it changes sign after every two kicks [i.e., $f(n)=1$ if $n=4 j+1$, or $4 j+2$, and $f(n)=-1$ if $n$ $=4 j+3$ or $4 j+4$, where $j$ is an integer], $\hat{L}$ is the angular momentum operator, $\theta$ is the conjugate angle, $I$ is the moment of inertia, $\lambda$ is the strength of the kicking field, and $T$ is the time interval between kicks. The basis states of their Hilbert spaces are given by $|m\rangle$, with $\hat{L}|m\rangle=m \hbar|m\rangle$. The quantum propagator in the $\mathrm{KR}$ case for times $\left(N-0^{+}\right) T$ to $\left(N+1-0^{+}\right) T$ is given by

$$
\hat{F}^{K R}(\tau, k)=\exp \left(i \frac{\tau}{2} \frac{\partial^{2}}{\partial \theta^{2}}\right) \exp [-i k \cos (\theta)],
$$

with dimensionless parameters $k=\lambda T / \hbar$ and the effective Planck constant $\tau=\hbar T / I$. The classical limit of the $\mathrm{KR}$ quantum map, i.e., the standard map, depends only on one parameter $\kappa \equiv k \tau$ and takes the following form:

$$
\begin{gathered}
\widetilde{L}_{N}=\widetilde{L}_{N-1}+\kappa \sin \left(\theta_{N-1}\right), \\
\theta_{N}=\theta_{N-1}+\widetilde{L}_{N}
\end{gathered}
$$

where $\widetilde{L} \equiv L \tau / \hbar$ is the scaled $c$-number angular momentum and $\left(\widetilde{L}_{N}, \theta_{N}\right)$ represents the phase space location of a classical trajectory at $\left(N+1-0^{+}\right) T$. With similar notation, the quantum map associated with $\mathrm{MKR}$, for times ranging from $\left(4 j+1-0^{+}\right) T$ to $\left[4(j+1)+1-0^{+}\right] T$, can be written as

$$
\hat{F}^{M K R}(\tau, k)=\left[\hat{F}^{K R}(\tau,-k)\right]^{2}\left[\hat{F}^{K R}(\tau, k)\right]^{2} .
$$

The classical limit of the MKR quantum map [Eq. (5)] is given by 

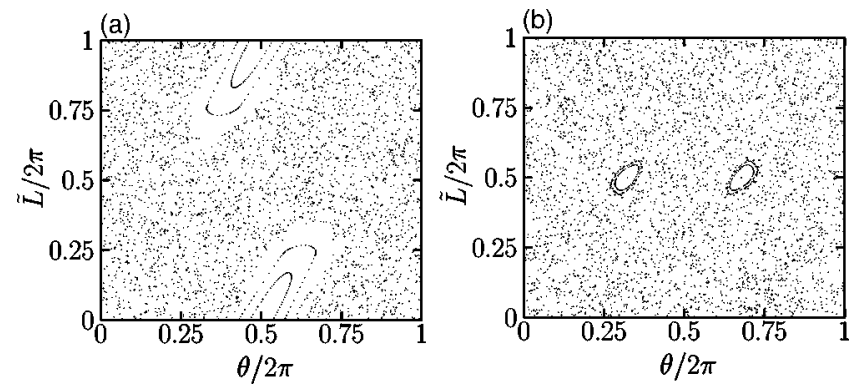

FIG. 1. Classical phase space structures for (a) the standard map and (b) the map of Eq. (6), in the case of $\kappa=3.5$. All variables are in dimensionless units. Note that the regular islands seen in (b) are transporting while those in (a) are not.

$$
\begin{gathered}
\widetilde{L}_{4 j+1}=\widetilde{L}_{4 j}+\kappa \sin \left(\theta_{4 j}\right), \quad \theta_{4 j+1}=\theta_{4 j}+\widetilde{L}_{4 j+1}, \\
\widetilde{L}_{4 j+2}=\widetilde{L}_{4 j+1}+\kappa \sin \left(\theta_{4 j+1}\right), \quad \theta_{4 j+2}=\theta_{4 j+1}+\widetilde{L}_{4 j+2}, \\
\widetilde{L}_{4 j+3}=\widetilde{L}_{4 j+2}-\kappa \sin \left(\theta_{4 j+2}\right), \quad \theta_{4 j+3}=\theta_{4 j+2}+\widetilde{L}_{4 j+3}, \\
\widetilde{L}_{4 j+4}=\widetilde{L}_{4 j+3}-\kappa \sin \left(\theta_{4 j+3}\right), \quad \theta_{4 j+4}=\theta_{4 j+3}+\widetilde{L}_{4 j+4} .
\end{gathered}
$$

The well-known accelerator modes in KR are closely related to the existence of the marginally stable points: $(\tilde{L}$ $=2 \pi l_{1}, \theta= \pm \pi / 2$ ) for $\kappa=2 \pi l_{2}$, where $l_{1}$ and $l_{2}$ are integers. These points are shifted by a constant value $\left( \pm 2 \pi l_{2}\right)$ in $\widetilde{L}$ after each iteration. For values of $\kappa$ close to $2 \pi l_{2}$, there still exist interesting transporting regular islands on which classical trajectories simply jump to other similar islands located in adjacent phase space cells, resulting in a ballistic increase of rotational energy. For trajectories initially outside the accelerator modes, the "stickiness" of the boundary between the accelerator modes and the chaotic sea induces anomalous diffusion over the energy space, i.e., energy increases in a nonlinear fashion, but not quadratically.

Examining the classical MKR map [Eq. (6)], we find a different type of marginally stable points. That is, for $\kappa$ $=\left(2 l_{2}+1\right) \pi$, trajectories emanating from $\widetilde{L}=\left(2 l_{1}+1\right) \pi, \theta$ $= \pm \pi / 2$ are shifted by a constant value $\left[ \pm\left(2 l_{2}+1\right) \pi\right]$ in $\widetilde{L}$ after each kick. As confirmed by our numerical experiments, this suggests that by reversing the kicking potential after every two kicks, one may create new transporting regular islands. For example, in Fig. 1 we show classical phase space structures of both $\mathrm{KR}$ and MKR for $\kappa=3.5$. The main regular islands seen in Fig. 1(a) in the KR case are not transporting, consistent with the fact that $\kappa=3.5$ is far from $2 \pi l_{2}$. By contrast, a simple computation reveals that those islands clearly seen in Fig. 1(b) in the MKR case are transporting (note that $\kappa=3.5$ is not far from $\pi$ ).

A number of additional remarks are in order:

(1) The transporting islands shown in Fig. 1(b) are much larger than the well-studied accelerator modes in KR. A rough estimate gives their area at least ten times larger than the accelerator modes in KR with $\kappa=6.908745$, and four times larger with $\kappa=6.476939$ [11].

(2) The transporting islands are stable with changes in $\kappa$, e.g., the $\kappa=3.4$ or $\kappa=3.6$ case gives transporting islands of similar size.

(3) Unlike the accelerator modes in KR, the transporting islands of MKR are far away from $\widetilde{L}=0$. This latter feature favors the observation of anomalous diffusion, insofar as any initial state with sufficiently low energy will not overlap with the transporting islands of MKR.

(4) After each kick, trajectories initiated from those islands shown in Fig. 1(b) will increase their $\widetilde{L}$ by $\pm \pi$ approximately, although the phase space structures have a period of $2 \pi$.

(5) There exist interesting extensions of MKR. For instance, consider the cases in which the kicking potential is reversed after every $N>2$ kicks where $N=6,10,14, \ldots$. Then, for $\kappa=\left(2 l_{2}+1\right) \pi$, trajectories emanating from $[\tilde{L}$ $\left.=\left(2 l_{1}+1\right) \pi, \theta= \pm \pi / 2\right]$ are also transporting, with their shift of angular momentum after each kick alternating between different constant values. However, we have found that the transporting islands associated with these generalized cases are extremely small and very sensitive to the value of $\kappa$.

Consider now quantum versus classical energy absorption behavior in each of KR and MKR. We choose $\kappa=3.5$ as an example; the results described below can be observed for a wide range of parameters $\kappa$ (e.g., for $3.3<\kappa<5.0$ ). We define the dimensionless scaled rotational energy averaged over the quantum or classical ensemble as $\widetilde{E}_{q} \equiv\left\langle\hat{L}^{2}\right\rangle \tau^{2} / 2 \hbar^{2}$ or $\widetilde{E}_{c} \equiv\left\langle\widetilde{L}^{2}\right\rangle / 2$. The effective Planck constant $\tau$ is first chosen to be 0.1 , a value far from the semiclassical limit but relatively small compared to the area of the transporting islands shown in Fig. 1(b). Further, this value of $\tau$ ensures that the nongeneric behavior associated with quantum resonances (i.e., $\tau$ $\left.=2 \pi l_{1} / l_{2}\right)$ is avoided. The initial quantum state here is chosen to be $|0\rangle$, which evidently does not overlap with the transporting islands. The corresponding classical initial state is given by $\widetilde{L}=0$ with $\theta$ uniformly distributed in $[0,2 \pi]$. Figure 2(a) displays a quantum-classical comparison in the KR case. Clearly, quantum effects in KR strongly suppress classical energy absorption and the QCC break time $t_{b}^{K R}$ in Fig. 2(a) is $\sim 20 T$. By contrast, Fig. 2(b) shows that both the quantum and classical MKR systems display characteristics of anomalous diffusion: an excellent log-log linear fit of the results in Fig. 2(b) after the MKR QCC break time $t_{b}^{M K R}$ [that is, we numerically fit the results with $\ln \left(\widetilde{E}_{q}\right)=a \ln (N)$ $+b$ and $\ln \left(\widetilde{E}_{c}\right)=a^{\prime} \ln (N)+b^{\prime}$, where $a, b, a^{\prime}$, and $b^{\prime}$ are parameters] gives that $\widetilde{E}_{q} \propto N^{1.85}$ (solid line) and $\widetilde{E}_{c} \propto N^{1.36}$ (dashed line), where $N$ is the number of kicks. Hence, significantly, the quantum energy absorption is seen to be far greater than that associated with the underlying classical anomalous diffusion. This rapid quantum growth is even more visible in Fig. 2(c), which shows that the faster-thanclassical excitation process shown in Fig. 2(b) persists for much longer time scales, as well as for an effective Planck constant that is ten times smaller [see the discrete points in 
(a)

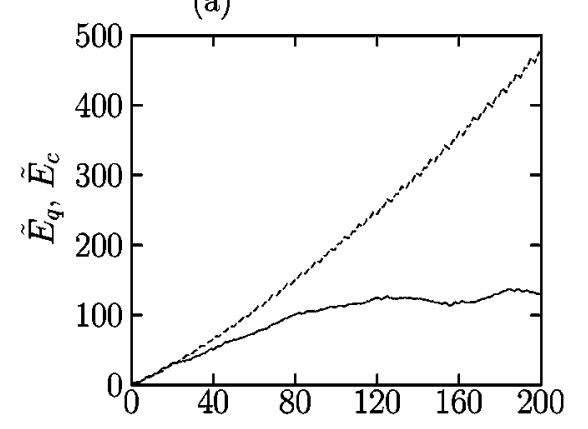

(b)

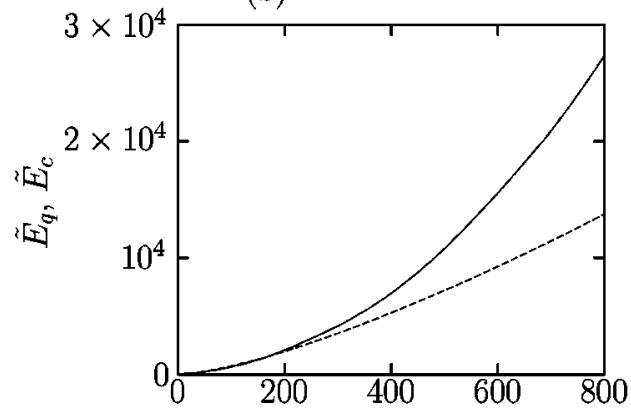

(c)

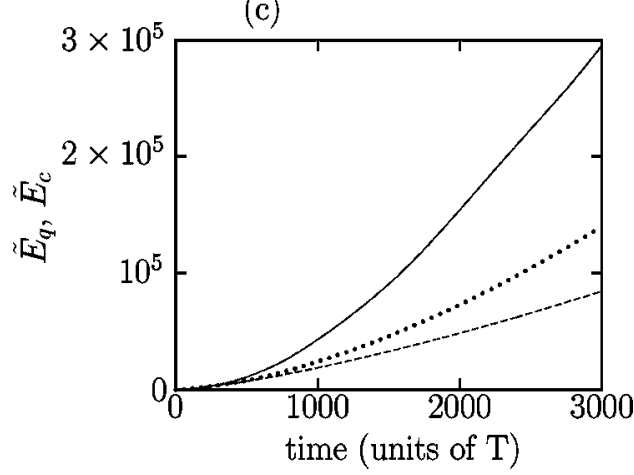

FIG. 2. The time dependence of the average dimensionless scaled rotational energy, denoted as $\widetilde{E}_{q}$ and $\widetilde{E}_{c}$ for quantum and classical ensembles, respectively, for (a) KR, (b) MKR, and (c) MKR over even longer time scales, with $\kappa=3.5$. Solid lines denote quantum results for $\tau=0.1$, and dashed lines denote classical results. The discrete points in (c) represent quantum results for $\tau$ $=0.01$. Note that in (b) and (c) quantum results are well above the classical results.

Fig. 2(c)]. Note also that in the MKR case shown in Fig. 2(b), quantum dynamics agrees fairly well with the classical result for up to $t_{b}^{M K R} \sim 200 T$, much longer than $t_{b}^{K R} \sim 20 T$ in Fig. 2(a). In addition, $t_{b}^{M K R}$ is also an order of magnitude larger than the characteristic QCC time scale found in the $\mathrm{KR}$ model in the presence of accelerator modes with the same effective Planck constant [8]. This is understandable because the main transporting regular islands of MKR are larger than the accelerator modes of KR, and therefore classical phase space structures can be "seen" more clearly by the quantized MKR.

Also evident in Fig. 2(c) is that a smaller effective Planck constant gives better QCC. Thus, in contrast to what was suggested in Ref. [8], the quantum anomalous diffusion here

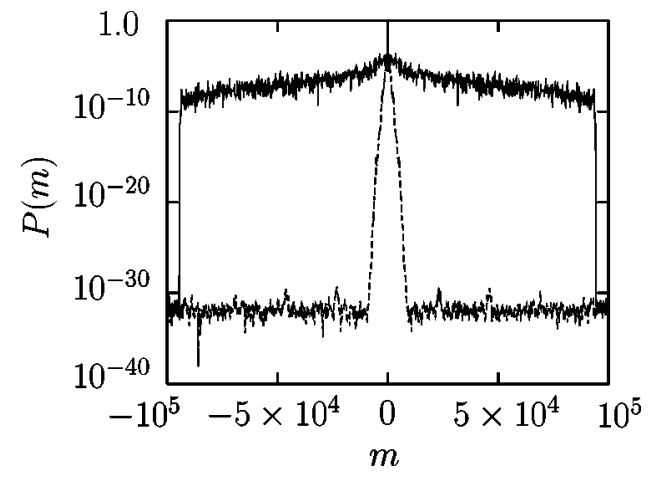

FIG. 3. A comparison between KR (dashed line) and MKR (solid line) in terms of the probability $P(m)$ of finding the system in the state $|m\rangle$ at $t=3000 T . \kappa=3.5, \tau=0.1$, and the initial state is $|0\rangle$. Fluctuations of $P(\mathrm{~m})$ below the $10^{-30}$ level are due to numerical cutoff errors.

approaches the underlying classical anomalous diffusion from above rather than from below as the effective Planck constant goes to zero. This leads to the conclusion that under nonresonant conditions quantization does not necessarily induce suppression of energy absorption. Note that one previous work [17] also reported computations (in the kickedHarper model) on faster-than-classical diffusion under nonresonant conditions. However, in Ref. [17] the growth in the second moment of angular momentum is always quadratic, and the issue of faster-than-classical energy absorption cannot be addressed since the kinetic energy therein is a cosine function of angular momentum. It should also be pointed out that, unlike gravity-induced quantum accelerator modes in KR [18], quantum anomalous diffusion in MKR is shown to have a well-defined classical limit and does not require the value of $\tau$ to be near quantum resonance conditions.

It is interesting to view MKR from a quantum control [16] perspective. The time-evolving wave function can be expanded as a superposition of different $|m\rangle$ states, i.e., $\Sigma_{m} C_{m}\langle\theta \mid m\rangle$, where $C_{m}$ are the expansion coefficients. Since $\cos (\theta+\pi)=-\cos (\theta) \quad$ and $\quad \Sigma C_{m}\langle\theta+\pi \mid m\rangle=\Sigma_{m}$ $(-1)^{m} C_{m}\langle\theta \mid m\rangle$, the effect of reversing the kicking potential is equivalent to adding a $\pi$ phase difference between all neighboring basis states. As such, the potential reversal in MKR can be regarded as periodically introducing quantum phases into KR and therefore as a significant extension of our previous coherent control work [13]. In addition, noting that

$$
\hat{F}^{M K R}(\tau, k)=\left[\exp \left(i \pi \frac{\partial^{2}}{\partial \theta^{2}}\right) \hat{F}^{K R}(\tau, k) \hat{F}^{K R}(\tau, k)\right]^{2},
$$

we have that MKR can be effectively realized by using KR and introducing a time delay $t_{d}=2 \pi T / \tau$ of the kicking field after every two kicks.

Figure 3 displays a comparison between KR and MKR in terms of the occupation probability $P(m)$ of $|m\rangle$ after 3000 kicks, with $\kappa=3.5, \tau=0.1$, and the initial state $|0\rangle$. It is seen that for many states (e.g., for $90000>|m|>8000$ ), $P(m)$ of MKR is greater than that of KR by a factor larger than $10^{20}$. 


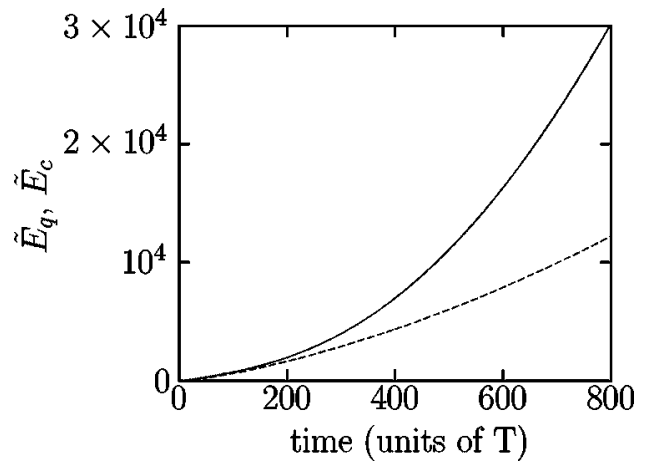

FIG. 4. As in Fig. 2(b), except the initial condition, the periodic boundary condition, the values of $\tau$ and $\kappa$ are all changed (see the text for details). The classical initial distribution function is chosen to be identical with the Wigner function of the initial quantum state.

Further, we find that $\widetilde{E}_{q}$ of MKR at $t=3000 T$ is three orders of magnitude larger than that of KR, whereas classical dynamics only gives an energy-absorption difference of $\sim 2.5$ times. We stress (1) that this vast quantum control over energy absorption is achieved by simply reversing the kicking potential, or, alternatively, by introducing a certain time delay after every two kicks, and (2) that the control mechanism here is uniquely based upon quantum anomalous diffusion in MKR, and is thus far more effective than that in amplitudemodulated $[19,20]$ or phase-modulated kicked-rotor systems [21] in the absence of transporting islands. We also note that if we regard MKR as controlled KR, then we see that we still have control for an effective Planck constant as large as $\tau$ $=1.0[21]$.

It remains to examine the possibility of observing fasterthan-classical quantum anomalous diffusion in atom optics experiments. In atom optics experiments on KR, the angular variable $\theta$ is replaced by the position variable of atoms [2] so that there is no periodic boundary condition as in $|\psi(\theta=0)\rangle=|\psi(\theta=2 \pi)\rangle$ in KR. Further, in the experiments, translational Gaussian states (which are different from the initial state that we have examined thus far) are typically used as initial states. Thus, of particular concern here is that the faster-than-classical quantum anomalous diffusion is found to be insensitive to the periodic boundary condition and to the initial conditions. That is, in sharp contrast to quantum resonances in KR, the faster-than-classical anomalous diffusion shown in Figs. 2(b) and 2(c) is found to be stable with respect to small variations of all system parameters and initial conditions. For example, Fig. 4 shows similar faster-than-classical anomalous diffusion with the arbi- trary choice of $\tau=2 \pi /(60+\sigma)$, where $\sigma=(\sqrt{5}-1) / 2, k$ $=33$, the initial state being given by a Gaussian $|\psi(\theta)\rangle$ $=\exp \left(-\theta^{2} / 18\right) / \sqrt{3} \pi^{1 / 4}$, and the periodic boundary condition changed from $|\psi(\theta=0)\rangle=|\psi(\theta=2 \pi)\rangle$ to $|\psi(\theta=0)\rangle$ $=|\psi(\theta=512 \pi)\rangle$. This kind of result suggests that fasterthan-classical quantum anomalous diffusion should be observable in atom optics experiments, provided that the relatively small effective Planck constant $\tau \sim 0.1$ is achieved.

We qualitatively explain faster-than-classical anomalous diffusion in terms of a quantum tunneling mechanism [11]. At early times, both the quantum and classical systems that are not initially on the transporting regular islands tend to be trapped by the fractal structures lying between the main transporting regular islands and the chaotic sea, resulting in excellent QCC in anomalous diffusion. Later, for $t \sim t_{b}^{M K R}$, a non-negligible part of the time-evolving quantum state has tunneled from the chaotic sea to the large transporting islands, whereas each classical trajectory can only sojourn in the neighborhood of the transporting islands for a certain time. Thus, if the mean sojourn time of classical trajectories is relatively short compared to the characteristic time scale over which the transporting-island component of the timeevolving quantum state builds up and then tunnels back to the chaotic sea, the quantum tunneling effects can strongly enhance the transport in phase space, and therefore quantum anomalous diffusion can be significantly faster than classical anomalous diffusion. This also makes it clear that quantum anomalous diffusion in MKR will slow down and dynamical localization can take place when significant tunneling in the reverse direction (i.e., from the transporting islands to the chaotic sea) occurs. Indeed, additional numerical results (not shown) for longer time scales (e.g., 12000 kicks in the case of $\tau=0.1$ ) and for larger effective Planck constants indicate that quantum anomalous diffusion in MKR will eventually be suppressed by dynamical localization, with a larger $\tau$ giving earlier suppression.

In conclusion, we have found large transporting regular islands in the classical phase space of a modified kickedrotor system, and have shown that the associated quantum anomalous diffusion can be significantly faster than classical anomalous diffusion over long time scales. The results are of interest to both areas of quantum chaos and quantum control.

This work was supported by the U.S. Office of Naval Research and the Natural Sciences and Engineering Research Council of Canada. H.J.W. was partially supported by the Studienstiftung des Deutschen Volkes and the Barth Fonds of ETH Zürich.
[1] G. Casati and B. Chirikov, Quantum Chaos: Between Order and Disorder (Cambridge University Press, New York, 1995).

[2] F.L. Moore et al., Phys. Rev. Lett. 75, 4598 (1995); H. Ammann et al., ibid. 80, 4111 (1998); J. Ringot et al., ibid. 85, 2741 (2000); M.B. d'Arcy et al., ibid. 87, 074102 (2001).

[3] R. Blümel, S. Fishman, and U. Smilansky, J. Chem. Phys. 84, 2604 (1986).
[4] I.Sh. Averbukh and R. Arvieu, Phys. Rev. Lett. 87, 163601 (2001).

[5] G. Casati, I. Guarneri, and G. Maspero, Phys. Rev. Lett. 84, 63 (1999).

[6] P. Facchi, S. Pascazio, and A. Scardicchio, Phys. Rev. Lett. 83, 61 (1999).

[7] B. Georgeot and D.L. Shepelyansky, Phys. Rev. Lett. 86, 2890 
(2001)

[8] R. Roncaglia et al., Phys. Rev. E 51, 5524 (1995).

[9] B.G. Klappauf et al., Phys. Rev. Lett. 81, 4044 (1998).

[10] B. Sundaram and G.M. Zaslavsky, Phys. Rev. E 59, 7231 (1999).

[11] A. Iomin, S. Fishman, and G. Zaslavsky, Phys. Rev. E 65, 036215 (2002).

[12] L. Hufnagel et al., Phys. Rev. Lett. 89, 154101 (2002).

[13] J. Gong and P. Brumer, Phys. Rev. Lett. 86, 1741 (2001); J. Chem. Phys. 115, 3590 (2001).

[14] J. Gong and P. Brumer, Phys. Rev. Lett. 88, 203001 (2002).

[15] Our modified kicked-rotor model can be regarded as a specific realization of the so-called generalized kicked-rotor model, which was first introduced, in the context of quantum antiresonance, by I. Dana, E. Eisenberg, and N. Shnerb, Phys. Rev.
E 54, 5948 (1996).

[16] M. Shapiro and P. Brumer, Principles of the Quantum Control of Molecular Processes (Wiley, New York, 2003); M. Shapiro and P. Brumer, Adv. At., Mol., Opt. Phys. 42, 287 (2000); S.A. Rice and M. Zhao, Optical Control of Molecular Dynamics (Wiley, New York, 2000).

[17] R. Lima and D.L. Shepelyansky, Phys. Rev. Lett. 67, 1377 (1991).

[18] S. Fishman, I. Guarneri, and L. Rebuzzini, Phys. Rev. Lett. 89, 084101 (2002); S. Schlunk et al., e-print physics/0212015.

[19] D.L. Shepelyansky, Physica D 8, 208 (1983).

[20] G. Casati, I. Guarner, and D.L. Shepelyansky, Phys. Rev. Lett. 62, 345 (1989).

[21] J. Gong, H.J. Wörner, and P. Brumer (unpublished). 